\title{
SUBHARMONIC DYNAMO ACTION IN THE ROBERTS FLOW
}

\author{
F. PLUNIAN ${ }^{\mathrm{a}, *}$ and K.-H. RÄDLER ${ }^{\mathrm{b}}$ \\ ${ }^{a}$ Laboratoires des Ecoulements Géophysiques et Industriels, B.P. 53, 38041 Grenoble Cedex 9,

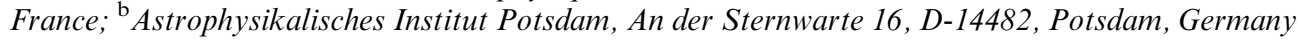

The paper deals with the dynamo action of the Roberts flow, that is, a flow depending periodically on two cartesian coordinates, $X$ and $Y$, but being independent of the third one, $Z$. In particular the case is considered in which the magnetic fields, which are periodic in $X, Y$ and $Z$, have period lengths in the $X Y$ plane being integer multiples of that of the flow. Two approaches are used. Firstly, the equations governing the magnetic field are reduced to a matrix eigenvalue problem, which is solved numerically. Secondly, a mean magnetic field is defined by averaging over proper areas in the $X Y$-plane, corresponding equations are derived, in which the induction effect of the flow occurs as an anisotropic $\alpha$-effect, and analytic solutions are given. The results are of particular interest for the Karlsruhe dynamo experiment, which works with a Roberts type flow consisting of 52 cells inside a cylindrical volume. In order to check the reliability of predictions concerning self-excitation based on the mean-field approach, analogous predictions are derived for a rectangular box containing 50 cells, and are compared with results obtained with the help of direct solutions of the eigenvalue problem mentioned. It turns out that the simple mean-field approach in general underestimates the requirements for self-excitation. The corresponding results agree with those obtained in the subharmonic approach only if the side length $L$ of the box, its height $H$ and the edge length $l$ of a spin generator satisfy $L \gg H \gg l$.

In Appendix $\mathrm{B}$, some comments on previous results concerning $\mathcal{A B C}$ dynamos are made in the light of the subharmonic formalism used in the paper.

Keywords: Dynamo effect; Roberts flow; Subharmonic solutions; Dynamo experiment; Mean-field theory

\section{INTRODUCTION}

It is widely believed that the magnetic fields of cosmic bodies are due to dynamo action. The kinematic approach to dynamo models consists in studying the induction equation for the magnetic field with a given motion. This equation reads

$$
\frac{\partial \mathbf{B}}{\partial t}=\nabla \times(\mathbf{U} \times \mathbf{B})+\eta \nabla^{2} \mathbf{B}, \quad \nabla \cdot \mathbf{B}=0,
$$

where $\eta$ means the magnetic diffusivity, $\mathbf{B}$ the magnetic field and $\mathbf{U}$ the fluid velocity. In the case of a steady flow we may expect solutions varying like $\exp (p t)$ in time, with the

*Corresponding author. E-mail: Franck.Plunian@hmg.inpg.fr 
real part of $p$ being the growth rate of the magnetic field considered. In this case, an eigenvalue problem for $\mathbf{B}$ with the eigenvalue parameter $p$ occurs.

Although the fluid motions in the cosmic objects are rather complex it is useful to consider also simple steady flow patterns which are periodic with respect to two or three cartesian coordinates. In this way we may find some understanding of the basic dynamo mechanisms. The domain of integration of (1) can then be restricted to one cell of such a pattern. In particular, flows possessing hyperbolic stagnation points and helicity have been investigated. Their analysis is based on the emergence of magnetic structures such as flux ropes and sheets, lying between the hyperbolic stagnation points of the flow (Childress, 1979).

A well-known example is the Roberts flow given by

$$
\mathbf{U}=U\left[\sin \frac{Y}{L_{U}}, \sin \frac{X}{L_{U}}, \chi\left(\cos \frac{X}{L_{U}}-\cos \frac{Y}{L_{U}}\right)\right]
$$

(Roberts, 1972), where $X, Y, Z$ are cartesian coordinates, $L_{U}$ and $U$ the characteristic length and speed of the flow. The coefficient $\chi$ is a parameter related to the helicity of the flow. The flow pattern is sketched in Fig. 1. When speaking in the following of a "cell" of this pattern we mean the smallest-possible region bounded by straight stream lines, e.g., that defined by $0 \leq Y-X \leq 2 \pi L_{U}$ and $0 \leq Y+X \leq 2 \pi L_{U}$. Neighboring cells have opposite flow directions. For later use we define the magnetic Reynolds number $R_{m}$ by

$$
R_{m}=\frac{U L_{U}}{\eta}
$$

Roberts (1972) demonstrated that flows of this kind are capable of dynamo action. In his numerical investigations, he considered magnetic fields with the same periodicity in $X$ and $Y$ as the flow pattern. Furthermore, he restricted attention on magnetic fields which have a mean part, that is, a non-zero average taken over an area corresponding

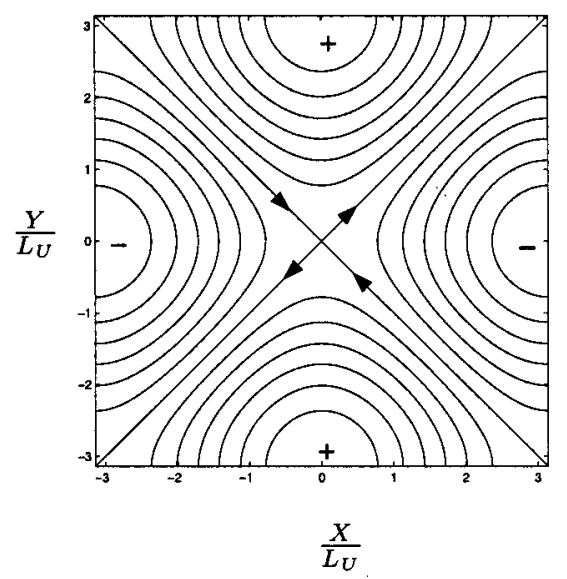

FIGURE 1 Stream lines of the Roberts flow in the $X Y$-plane. They coincide with isolines of the velocity in the $Z$-direction. 
to two cells, e.g., the period square shown in Fig. 1. This mean field does not depend on $X$ and $Y$ but depends on $Z$ and is therefore subject to dissipation. The overall mean (over $X, Y$ and $Z$ ) is zero. By this reason the dynamo considered is different from an externally excited one.

The dynamo action of the Roberts flow can be understood in terms of an anisotropic $\alpha$-effect with respect to the mean magnetic field. In the limit of large $R_{m}$, the order of magnitude of the $\alpha$-coefficient has been estimated (Childress, 1979) to be $\mathrm{O}\left(R_{m}^{-1 / 2}\right)$. Soward (1987) has shown that in this limit the dimensionless growth rate $\hat{p}=L_{U} p / U$ of the fastest mode satisfies $\hat{p}=\mathrm{O}\left[\ln \left(\ln R_{m}\right) / \ln R_{m}\right]$. That is, $\hat{p} \rightarrow 0$ as $R_{m} \rightarrow \infty$. So the Roberts dynamo proves to be a slow one. Nevertheless it shows a great efficiency (see also Soward, 1989, 1990, 1994; Childress and Gilbert, 1995).

In the Forschungszentrum Karlsruhe a dynamo experiment has been carried out with a flow of liquid sodium similar to the Roberts flow inside a cylindrical container (Müller and Stieglitz, 2000; Stieglitz and Müller, 2001). This has renewed the interest in the Roberts dynamo (see Busse et al., 1996; Rädler et al., 1996, 1997a,b, 1998; Tilgner, 1997).

Tilgner and Busse (1995) considered the case in which the period lengths of the magnetic field in the $X$ and $Y$ directions are integer multiples of the period length of the flow. They presented, however, numerical results for a few selected examples of such magnetic fields only.

In this paper, we deal in some more detail with subharmonic solutions of the Roberts dynamo problem for which the period length $L_{B}$ of the magnetic field is an integer multiple of the period length $L_{B}$ of the flow, that is

$$
\frac{L_{B}}{L_{U}}=N, \quad \text { with an integer } N \geq 1
$$

We further consider a mean-field approach to the Roberts dynamo problem, apply it for an estimate of the self-excitation condition in the Karlsruhe experiment and compare this with estimates gained with the help of subharmonic solutions (Section 3). Finally we discuss conclusions for the experiment (Section 4).

\section{SUBHARMONIC SOLUTIONS OF THE ROBERTS DYNAMO PROBLEM}

\subsection{Reduction of the Basic Equation}

We focus our attention on the induction Eq. (1) governing the magnetic field $\mathbf{B}$ in all space. Concerning the fluid velocity $\mathbf{U}$ we assume at first only that it is periodic with respect to $X$ and $Y$ with a period length $2 \pi L_{U}$ and independent of $Z$. We look for solutions B periodic in $X$ and $Y$ with a period length $2 \pi L_{B}$ so that (4) applies, and periodic in $Z$ with any period length.

In order to justify a proper representation for $\mathbf{B}$ we express for a moment the coordinates $X, Y$ and $Z$, shortly denoted by $\mathbf{X}$, according to $\mathbf{X}=\hat{\mathbf{x}} L_{B}$ by dimensionless coordinates $\hat{\mathbf{x}}$. Then these solutions can be represented as series of Fourier modes proportional to $\exp \{i \mathbf{K} \cdot \hat{\mathbf{x}}\}$, where $K_{x}$ and $K_{y}$ are integers but $K_{z}$ is an arbitrary real constant. The corresponding representation of $\mathbf{U}$, however, contains then only modes proportional to $\exp \left\{i N \mathbf{K}^{\prime} \cdot \hat{\mathbf{x}}\right\}$, where $K_{x}^{\prime}$ and $K_{y}^{\prime}$ are again integers but $K_{z}^{\prime}=0$. 
Considering Eq. (1) in view of the mode couplings caused by the $\mathbf{U} \times \mathbf{B}$ term, we see immediately that there is a set of independent solutions $\mathbf{B}$, each of which corresponds to a fixed vector $\mathbf{F}$ and is a series of modes proportional to $\exp \left\{i\left(\mathbf{F}+N \mathbf{K}^{\prime}\right) \cdot \hat{\mathbf{x}}\right\}$. Here $F_{x}$ and $F_{y}$ are integers, called $a$ and $b$ in the following and satisfying

$$
-N / 2<a, b \leq N / 2,
$$

$F_{z}$ is an arbitrary real constant, and $\mathbf{K}^{\prime}$ is again to be understood in the above sense.

In the following we shall use other dimensionless coordinates $\mathbf{x}$ instead of $\hat{\mathbf{x}}$, defined by $\mathbf{X}=\mathbf{x} L_{U}$, or $\hat{\mathbf{x}}=\mathbf{x} / N$. Then the last-mentioned modes are described by $\exp \left\{i\left(\mathbf{f}+\mathbf{K}^{\prime}\right) \cdot \mathbf{x}\right\}$ with $\mathbf{f}=\mathbf{F} / N$. In this way we see that all solutions $\mathbf{B}$ of (1) with the periodicity described above can be represented in the form

$$
\mathbf{B}=\operatorname{Re}\left[\mathrm{e}^{i \mathbf{f} \cdot \mathbf{x}} \mathbf{b}(\mathbf{x}, t)\right],
$$

where $\mathbf{f}$ is a vector given by

$$
\mathbf{f}=(a / N, b / N, k)
$$

with integers $a$ and $b$ satisfying (5) and $k$ being the dimensionless wave number for the $z$-direction. Further $\mathbf{b}$ is a vector field depending on $x$ and $y$ only and being periodic in both of them with the period length $2 \pi$, or in $X$ and $Y$ with $2 \pi L_{U}$. Of course, b depends on $\mathbf{f}$, too. In Table I the possible choices of $a / N$ and $b / N$ are given for $N$ up to 10 .

We note that for any given $\mathbf{f}$ several independent solutions of type (6) may exist. Obviously the solutions with $a=b=0$ coincide for all $N$, that is, are in fact the solutions with $N=1$ already investigated by Roberts. For $N=1$ there is, of course, no other choice of $a$ and $b$ than $a=b=0$. Furthermore, solutions (6) for a given set $a$, $b, N$ coincide with those for which this set is replaced by $n a, n b, n N$ with any integer $n$ (see also Table I). We finally point out that solutions with $a=0$ or $b=0$ possessing a non-zero mean in the above sense (depending on $z$ only) have, despite finite $L_{B}$, infinite length scales in $X$ or $Y$ directions, respectively.

Returning to Eq. (1), we put

$$
\mathbf{U}=U \mathbf{u}
$$

and introduce so the dimensionless velocity field $\mathbf{u}$, replace $\mathbf{X}$ by $\mathbf{x} L_{U}$ and $t$ by $t L_{U} / U$ with a new $t$, which is now dimensionless. Inserting then (6), we find

$$
\begin{gathered}
\frac{\partial \mathbf{b}}{\partial t}=\nabla \times(\mathbf{u} \times \mathbf{b})+i \mathbf{f} \times(\mathbf{u} \times \mathbf{b})+R_{m}^{-1}\left[\nabla^{2} \mathbf{b}+2 i(\mathbf{f} \cdot \nabla) \mathbf{b}-|\mathbf{f}|^{2} \mathbf{b}\right], \\
\nabla \cdot \mathbf{b}+i \mathbf{b} \cdot \mathbf{f}=0 .
\end{gathered}
$$

Using (10), we can rewrite (9) into ${ }^{1}$

$$
\frac{\partial \mathbf{b}}{\partial t}+(\mathbf{u} \cdot \nabla) \mathbf{b}=(\mathbf{b} \cdot \nabla) \mathbf{u}-i(\mathbf{f} \cdot \mathbf{u}) \mathbf{b}+R_{m}^{-1}\left[\nabla^{2} \mathbf{b}+2 i(\mathbf{f} \cdot \nabla) \mathbf{b}-|\mathbf{f}|^{2} \mathbf{b}\right] .
$$

\footnotetext{
${ }^{1}$ This equation has been already derived by Roberts (1972) but he used it only in the simplest case $a=b=0$ in its numerical calculations.
} 
TABLE I The possible choices of $(a / N, b / N)$ with $0 \leq a \leq b$ for $N$ up to 10

\begin{tabular}{|c|c|c|c|c|c|c|c|c|c|c|}
\hline$N$ & 1 & 2 & 3 & 4 & 5 & 6 & 7 & 8 & 9 & 10 \\
\hline & \multirow[t]{21}{*}{$(0,0)$} & $(0,0)$ & $(0,0)$ & $(0,0)$ & $(0,0)$ & $(0,0)$ & $(0,0)$ & $(0,0)$ & $(0,0)$ & $(0,0)$ \\
\hline & & $\left(0, \frac{1}{2}\right)$ & $\left(0, \frac{1}{3}\right)$ & $\left(0, \frac{1}{4}\right)$ & $\left(0, \frac{1}{5}\right)$ & $\left(0, \frac{1}{6}\right)$ & $\left(0, \frac{1}{7}\right)$ & $\left(0, \frac{1}{8}\right)$ & $\left(0, \frac{1}{9}\right)$ & $\left(0, \frac{1}{10}\right)$ \\
\hline & & $\left(\frac{1}{2}, \frac{1}{2}\right)$ & $\left(\frac{1}{3}, \frac{1}{3}\right)$ & $\left(\frac{1}{4}, \frac{1}{4}\right)$ & $\left(\frac{1}{5}, \frac{1}{5}\right)$ & $\left(\frac{1}{6}, \frac{1}{6}\right)$ & $\left(\frac{1}{7}, \frac{1}{7}\right)$ & $\left(\frac{1}{8}, \frac{1}{8}\right)$ & $\left(\frac{1}{9}, \frac{1}{9}\right)$ & $\left(\frac{1}{10}, \frac{1}{10}\right)$ \\
\hline & & & & $\left(0, \frac{1}{2}\right)$ & $\left(0, \frac{2}{5}\right)$ & $\left(0, \frac{1}{3}\right)$ & $\left(0, \frac{2}{7}\right)$ & $\left(0, \frac{1}{4}\right)$ & $\left(0, \frac{2}{9}\right)$ & $\left(0, \frac{1}{5}\right)$ \\
\hline & & & & $\left(\frac{1}{4}, \frac{1}{2}\right)$ & $\left(\frac{1}{5}, \frac{2}{5}\right)$ & $\left(\frac{1}{6}, \frac{1}{3}\right)$ & $\left(\frac{1}{7}, \frac{2}{7}\right)$ & $\left(\frac{1}{8}, \frac{1}{4}\right)$ & $\left(\frac{1}{9}, \frac{2}{9}\right)$ & $\left(\frac{1}{10}, \frac{1}{5}\right)$ \\
\hline & & & & $\left(\frac{1}{2}, \frac{1}{2}\right)$ & $\left(\frac{2}{5}, \frac{2}{5}\right)$ & $\left(\frac{1}{3}, \frac{1}{3}\right)$ & $\left(\frac{2}{7}, \frac{2}{7}\right)$ & $\left(\frac{1}{4}, \frac{1}{4}\right)$ & $\left(\frac{2}{9}, \frac{2}{9}\right)$ & $\left(\frac{1}{5}, \frac{1}{5}\right)$ \\
\hline & & & & & & $\left(0, \frac{1}{2}\right)$ & $\left(0, \frac{3}{7}\right)$ & $\left(0, \frac{3}{8}\right)$ & $\left(0, \frac{1}{3}\right)$ & $\left(0, \frac{3}{10}\right)$ \\
\hline & & & & & & $\left(\frac{1}{6}, \frac{1}{2}\right)$ & $\left(\frac{1}{7}, \frac{3}{7}\right)$ & $\left(\frac{1}{8}, \frac{3}{8}\right)$ & $\left(\frac{1}{9}, \frac{3}{9}\right)$ & $\left(\frac{1}{10}, \frac{3}{10}\right)$ \\
\hline & & & & & & $\left(\frac{1}{3}, \frac{1}{2}\right)$ & $\left(\frac{2}{7}, \frac{3}{7}\right)$ & $\left(\frac{1}{4}, \frac{3}{8}\right)$ & $\left(\frac{2}{9}, \frac{1}{3}\right)$ & $\left(\frac{1}{5}, \frac{3}{10}\right)$ \\
\hline & & & & & & $\left(\frac{1}{2}, \frac{1}{2}\right)$ & $\left(\frac{3}{7}, \frac{3}{7}\right)$ & $\left(\frac{3}{8}, \frac{3}{8}\right)$ & $\left(\frac{1}{3}, \frac{1}{3}\right)$ & $\left(\frac{3}{10}, \frac{3}{10}\right)$ \\
\hline & & & & & & & & $\left(0, \frac{1}{2}\right)$ & $\left(0, \frac{4}{9}\right)$ & $\left(0, \frac{2}{5}\right)$ \\
\hline & & & & & & & & $\left(\frac{1}{8}, \frac{1}{2}\right)$ & $\left(\frac{1}{9}, \frac{4}{9}\right)$ & $\left(\frac{1}{10}, \frac{2}{5}\right)$ \\
\hline & & & & & & & & $\left(\frac{1}{4}, \frac{1}{2}\right)$ & $\left(\frac{2}{9}, \frac{4}{9}\right)$ & $\left(\frac{1}{5}, \frac{2}{5}\right)$ \\
\hline & & & & & & & & $\left(\frac{3}{8}, \frac{1}{2}\right)$ & $\left(\frac{1}{3}, \frac{4}{9}\right)$ & $\left(\frac{3}{10}, \frac{2}{5}\right)$ \\
\hline & & & & & & & & $\left(\frac{1}{2}, \frac{1}{2}\right)$ & $\left(\frac{4}{9}, \frac{4}{9}\right)$ & $\left(\frac{2}{5}, \frac{2}{5}\right)$ \\
\hline & & & & & & & & & & $\left(0, \frac{1}{2}\right)$ \\
\hline & & & & & & & & & & $\left(\frac{1}{10}, \frac{1}{2}\right)$ \\
\hline & & & & & & & & & & $\left(\frac{1}{5}, \frac{1}{2}\right)$ \\
\hline & & & & & & & & & & $\left(\frac{3}{10}, \frac{1}{2}\right)$ \\
\hline & & & & & & & & & & $\left(\frac{2}{5}, \frac{1}{2}\right)$ \\
\hline & & & & & & & & & & $\left(\frac{1}{2}, \frac{1}{2}\right)$ \\
\hline
\end{tabular}

The $x$ and $y$ components of the last equation contain $b_{x}$ and $b_{y}$ only but not $b_{z}$. With

$$
\left(b_{x}, b_{y}\right)=\sum_{m, n}\left(g_{m, n}, h_{m, n}\right) \exp \{i(m x+n y)+p t\}
$$

where $p$ is a complex quantity, they turn into

$$
\begin{aligned}
(p+ & \left.R_{m}^{-1}\left[\left(m+f_{x}\right)^{2}+\left(n+f_{y}\right)^{2}+k^{2}\right]\right) \mathbf{b}_{m, n} \\
= & -\frac{1}{2} i k \chi\left(\mathbf{b}_{m+1, n}+\mathbf{b}_{m-1, n}-\mathbf{b}_{m, n+1}-\mathbf{b}_{m, n-1}\right) \\
& +\frac{1}{2}\left(m+f_{x}\right)\left(\mathbf{b}_{m, n+1}-\mathbf{b}_{m, n-1}\right)+\frac{1}{2}\left(n+f_{y}\right)\left(\mathbf{b}_{m+1, n}-\mathbf{b}_{m-1, n}\right) \\
& +\frac{1}{2}\left(h_{m, n+1}+h_{m, n-1}, g_{m+1, n}+g_{m-1, n}\right) .
\end{aligned}
$$

The Eq. (12) define a matrix eigenvalue problem with $p$ being the eigenvalue parameter. After solving it, we know $b_{x}$ and $b_{y}$, and we can calculate $b_{z}$ from (10) without any 
integration. The real part of $p$ gives the growth rate of the magnetic field. If there is a non-zero imaginary part of $p$ the magnetic field migrates in $Z$ direction.

We are in particular interested in the growth rates of the various solutions $\mathbf{B}$. Due to the symmetries of the flow the growth rates of different solutions coincide. As a rotation of the flow pattern by $\pi / 2$ about the $z$-axis changes only the signs of all velocity components, and reflections at the plane $x=0$ or $y=0$ change only the sign of the components in the $x y$-plane, and so the sign of the helicity, the growth rate remains unchanged under these transformations. In order to obtain the full spectrum of growth rates of solutions with a given $N$, it is thus sufficient to investigate the solutions with $0 \leq a \leq b \leq N / 2$ (see Table I). For $N=2 q$ as well as for $N=2 q+1$ the number of cases to be investigated is $(q+1)(q+2) / 2$. In addition, starting from solutions with $k>0$, solutions with $k<0$ can be obtained by reflection at $z=0$, under which again only the sign of the helicity is changed. Solutions obtained by such a reflection, too, coincide in $p$. Therefore, it is sufficient to consider only positive values of $k$.

\subsection{Numerical Results}

The eigenvalue problem defined by (12) has been solved numerically using the subroutine CGEEV of the standard LA PACK driver routine (version 1.0).

In Fig. 2 the growth rates $\operatorname{Re}(p)$ for $\chi=1, R_{m}=16$ and both $N=8$ and $N=10$ are plotted versus $k$. For each type of solutions with given $a$ and $b$ only that with the largest growth rate is considered. All these solutions have a non-zero mean part in the sense explained above, that is, as an average over an area corresponding to two cells of the flow pattern. The solution with $a=b=0$ (depending on $z$ only) is dominant for all $k$, that is, shows the largest growth rate when compared to the other solutions. Like this solution also those with $a=0$ and $b=1$, as well as those with $a=1$ and $b=0$, have infinite length scales. The fastest-growing solution with finite length scales is, apart from a range of small $k$, that with $a=b=1$.

Tilgner and Busse (1995) presented results like those given in Fig. 2, but for $R_{m}=15$ and only with $a / N=0,0.5$ and $b / N=0,0.5$. They are in good agreement with our results.

Let us focus our attention on the parameter range which is of interest for the Karlsruhe dynamo experiment, that is, $R_{m}$ in the order of unity, $\chi$ between 0 and 10 , $N$ around 10, and $k$ close to $1 / 5$ (see Section 3.3).

In Fig. 3 the growth rates $\operatorname{Re}(p)$ for several such values of $R_{m}$ and $k=1 / 5$ are plotted versus $\chi$. Among the non-decaying solutions with finite length scales always that with $a=b=1$ is dominant. Only with decaying solutions a dominance of such with other $a$ and $b$ has been observed for $R_{m} \leq 1$ and $\chi \geq 6$.

In Fig. 4 marginal values of $R_{m}$ are plotted versus $k$ or, in other words, the ranges of dynamo activity in the $R_{m} k$-plane are given for $\chi=1$ and various values of $f=a / N=b / N$. For $R_{m}$ up to 60 , the range for $f=1 / 10$ contains those for larger $f$. In order to determine the marginal curve for finite length scales solutions in the case $N=10$ it is therefore sufficient to consider the solution with $a=b=1$. The curve for $f=0$ is again given for comparison only.

Here we open a parenthesis to report the results of further calculations of the solution $a=b=0$ for larger $R_{m}$. For this purpose it is sufficient to adopt $N=1$. As mentioned by Roberts (1972), due to the symmetries of the flow, there are four independent solutions. Only two of them have non-zero mean parts. They have been studied by 

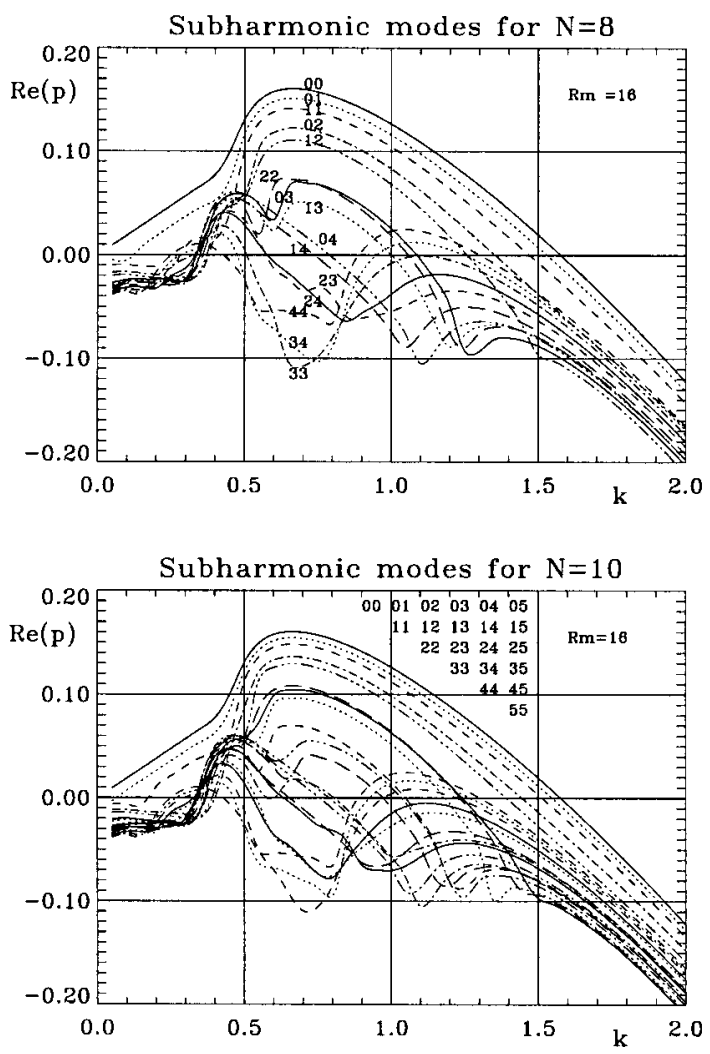

FIGURE 2 Growth rates $\operatorname{Re}(p)$ versus $k$, for $\chi=1, R_{m}=16$ and both $N=8$ (top) and $N=10$ (bottom). The labels give the values of $a$ and $b$ (cf. Table I). Note that the results for $a=b=0$ apply to arbitrary $N$. Labels for $N=10$ are not at the curves for the sake of clarity.

Roberts (1972) for $R_{m} \leq 64$ and by Soward (1987) in the asymptotic limit of large $R_{m}$. However, it is not a priori obvious that these solutions are always dominant in the limit of large $R_{m}$ (see, e.g., Plunian et al., 1999). This is why we made further calculations for $R_{m}$ up to $2^{11}$, looking for all four solutions. It appears that the solution with largest growth rate always has a non-zero mean. For $R_{m} \geq 2^{9}$ there is indeed a growing zero-mean solution with positive growth rate but the latter is by a factor 10 below the growth rate of the dominant solution. As a result, the assumption of non-zero mean made by Soward (1987) for asymptotic estimate of $p$ in the limit of large $R_{m}$ seems indeed to be the relevant one.

\section{MEAN-FIELD APPROACH TO THE ROBERTS DYNAMO PROBLEM AND ITS APPLICATION TO THE KARLSRUHE EXPERIMENT}

\subsection{The Mean-Field Approach}

Following the lines of mean-field dynamo theory as it is often used in astrophysical context (see, e.g., Krause and Rädler, 1980), a mean-field approach has been developed to 


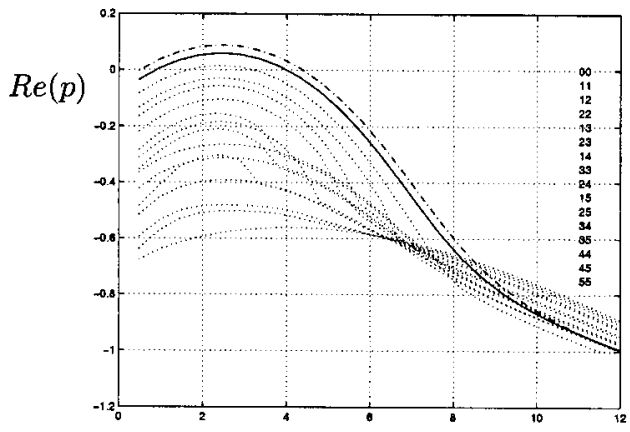

(a)

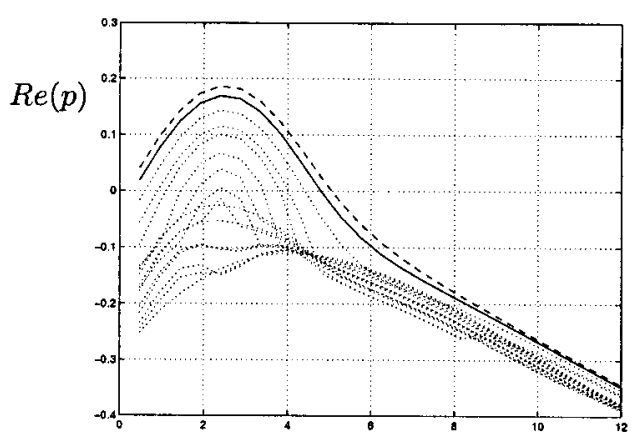

(c)

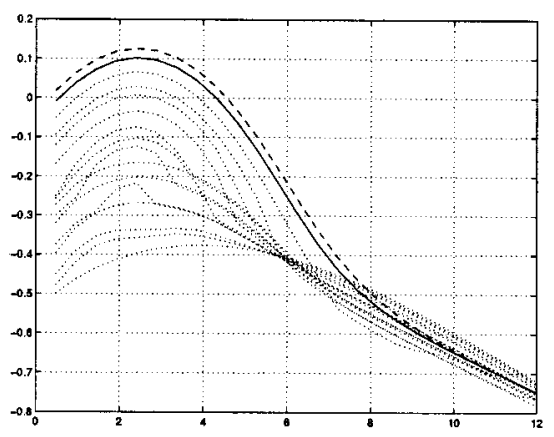

(b)

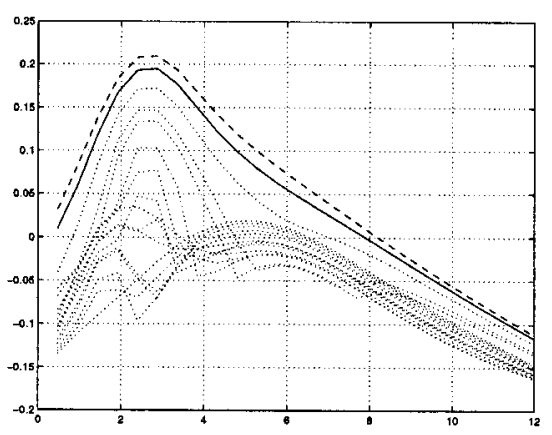

(d) $\chi$

FIGURE 3 Growth rates $\operatorname{Re}(p)$ versus $\chi$, for $k=1 / 5$ and (a) $R_{m}=0.75$, (b) $R_{m}=1$, (c) $R_{m}=2$ and (d) $R_{m}=4$. The dashed curves correspond to the solutions with $a=b=0$, the solid curves to those with $a=b=1$ and $N=10$, and the dotted curves to the other solutions with finite length scales with $a / N$ and $b / N$ as given for $N=10$ in Table I. The labels $a b$ on the right of (a) belong in this order to the left ends of the curves. In (b), (c) and (d) the first eight of these labels have to be assigned to the eight upper left ends of the curves.

the Roberts dynamo problem (see Rädler et al., 1996, 1997a, 1998). In this approach both the magnetic field $\mathbf{B}$ and the fluid velocity $\mathbf{U}$ are understood as sums of mean fields, $\overline{\mathbf{B}}$ and $\overline{\mathbf{U}}$, and fluctuating fields, $\mathbf{B}^{\prime}$ and $\mathbf{U}^{\prime}$. A mean field is defined by averaging the original field over an area corresponding to two cells in the $X Y$-plane, for example

$$
\overline{\mathbf{B}}(X, Y, Z, t)=\int_{-\pi L_{U}}^{+\pi L_{U}} \int_{-\pi L_{U}}^{+\pi L_{U}} \mathbf{B}(X+\xi, Y+\zeta, Z, t) d \xi d \zeta
$$

Clearly, we have $\overline{\mathbf{U}}=\mathbf{0}$. Subjecting the induction Eq. (1) to this kind of averaging we obtain

$$
\frac{\partial \overline{\mathbf{B}}}{\partial t}=\nabla \times \mathcal{E}+\eta \nabla^{2} \overline{\mathbf{B}}, \quad \nabla \cdot \overline{\mathbf{B}}=0
$$




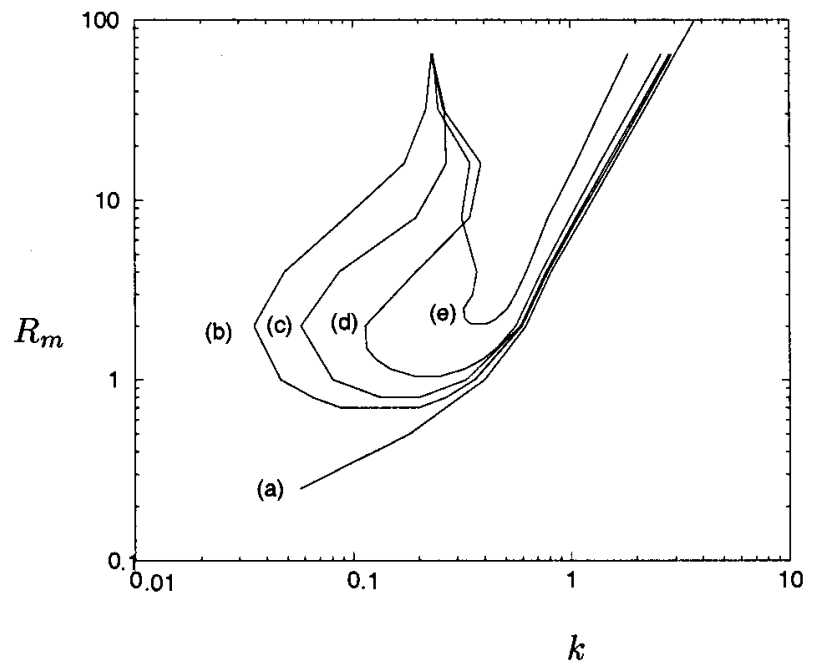

FIGURE 4 Marginal values of $R_{m}$ versus $k$ for $\chi=1$ and various $f=a / N=b / N$. Curve (a) belongs to $f=0$, (b) to $f=1 / 10$, (c) to $f=1 / 8$, (d) to $f=1 / 6$, (e) to $f=1 / 4$.

where $\mathcal{E}$ is a mean electromotive force due to the fluid motions given by

$$
\mathcal{E}=\overline{\mathbf{U} \times \mathbf{B}}
$$

We may consider $\mathcal{E}$ as a functional of $\mathbf{U}$ and $\overline{\mathbf{B}}$. Let us accept the assumption usually adopted in the mean-field context that $\mathcal{E}$ in a given point in space and time depends on $\overline{\mathbf{B}}$ only via the components of $\overline{\mathbf{B}}$ and their first spatial derivatives in this point. This is reasonable for sufficiently small variations of $\overline{\mathbf{B}}$ in space and time. Then we may conclude from the symmetry properties of the Roberts flow that

$$
\begin{aligned}
\mathcal{E}= & -\alpha_{\perp}[\overline{\mathbf{B}}-(\hat{\mathbf{Z}} \cdot \overline{\mathbf{B}}) \hat{\mathbf{Z}}]-\beta_{\perp} \nabla \times \overline{\mathbf{B}}-\left(\beta_{\|}-\beta_{\perp}\right)(\hat{\mathbf{Z}} \cdot \nabla \times \overline{\mathbf{B}}) \hat{\mathbf{Z}} \\
& -\beta_{3} \hat{\mathbf{Z}} \times[\nabla(\hat{\mathbf{Z}} \cdot \overline{\mathbf{B}})+(\hat{\mathbf{Z}} \cdot \nabla) \overline{\mathbf{B}}],
\end{aligned}
$$

(see also Rädler et al., 1996), where $\alpha_{\perp}, \beta_{\perp}, \beta_{\|}$and $\beta_{3}$ are constants determined by $U, \chi, L_{U}$ and $\eta$, and $\hat{\mathbf{Z}}$ is the unit vector in $Z$-direction. The term with $\alpha_{\perp}$ describes an anisotropic $\alpha$-effect, acting in the $X Y$-plane only. The terms with $\beta_{\perp}$ and $\beta_{\|}$can be interpreted by introducing a mean-field conductivity different from the conductivity involved in $\eta$ and being anisotropic. Finally, the term with $\beta_{3}$ describes a part of $\mathcal{E}$ depending on the derivatives of $\overline{\mathbf{B}}$, which cannot be expressed by $\nabla \times \overline{\mathbf{B}}$, and therefore not interpreted in the sense of a modified conductivity.

For a first approximation, on which we restrict ourselves here, we consider no other contributions to $\mathcal{E}$ than that describing the anisotropic $\alpha$-effect, that is, we ignore all containing derivatives of $\overline{\mathbf{B}}$. Then we conclude from (14) and (16) that

$$
\frac{\partial \overline{\mathbf{B}}}{\partial t}=-\nabla \times\left\{\alpha_{\perp}[\overline{\mathbf{B}}-(\hat{\mathbf{Z}} \cdot \overline{\mathbf{B}}) \hat{\mathbf{Z}}]\right\}+\eta \nabla^{2} \overline{\mathbf{B}}, \quad \nabla \cdot \overline{\mathbf{B}}=0
$$


Several calculations of $\alpha_{\perp}$ have been carried for the Roberts flow (see Rädler et al., 1996, 1997a,b, 1998). In our notation the result reads

$$
\alpha_{\perp}= \pm \frac{\pi}{16 \sqrt{2}} \frac{\eta}{L_{U}} R_{m \|} R_{m \perp} \phi\left(R_{m \perp}\right)
$$

(see Appendix). The sign of $\alpha_{\perp}$ agrees with that of the helicity, that is, the upper sign applies to $\chi>0$, the lower to $\chi<0 . R_{m \perp}$ and $R_{m \|}$ are magnetic Reynolds numbers for the flow in the $X Y$-plane and in $Z$-direction, respectively, which are connected to $R_{m}$ introduced with (3) by

$$
R_{m \|}=\frac{8 \sqrt{2}}{\pi} \chi R_{m}, \quad R_{m \perp}=2 R_{m} .
$$

Furthermore, $\phi$ is a function satisfying $\phi(0)=1$ and monotonously decreasing to zero with growing $R_{m \perp}$. It is given in detail, e.g., in Rädler et al. (1996, 1997a,b, 1998, 2001).

\subsection{A Simple Mean-Field Theory of the Experiment}

The essential piece of the Karlsruhe dynamo experiment (see, e.g., Müller and Stieglitz, 2000; Stieglitz and Müller, 2001) is the "dynamo module", a cylindrical container with both radius and height somewhat less than $1 \mathrm{~m}$, through which liquid sodium is driven by external pumps. By means of system of channels with conducting walls, constituting 52 "spin generators", a helical motion is organized. The flow pattern is of Roberts type, with 52 cells in the sense explained above.

A simple estimate of the self-excitation condition for this experimental device has been derived from a special solution $\overline{\mathbf{B}}$ of the mean-field Eq. (17) with $\alpha_{\perp}$ assumed to be constant in all infinite space. There is in particular an axisymmetric solution for which special cylindrical surfaces around the symmetry axis and special planes perpendicular to it are not intersected by electric currents. The self-excitation condition for this solution, with the smallest of these cylindrical surfaces and two neighboring planes of this type coinciding with the surface of the dynamo module, was interpreted just as the excitation condition for the experimental device. It reads

$$
\left|\alpha_{\perp}\right| \geq \pi \frac{\eta}{H}\left[1+\left(\frac{3.83}{\pi}\right)^{2}\left(\frac{H}{R}\right)^{2}\right]
$$

where $R$ and $H$ are radius and height of the cylinder (see, e.g., Rädler et al., 1996).

This relation is by several reasons only a rough approximation. Apart from the neglect of all induction effects other than the $\alpha$-effect, the solution used does not satisfy realistic conditions to be required at the boundary of the dynamo module. In addition, it corresponds to the limit of a large number of cells inside the dynamo module. Finally, other investigations have shown that a magnetic field with a non-axisymmetric mean part can be excited more easily, that is, with lower values of $\left|\alpha_{\perp}\right|$, than that with an axisymmetric mean part assumed for the estimate (see, e.g., Rädler et al., 1996, 1997a,b, 1998). 
In the Karlsruhe experiment self-excitation has been indeed achieved with flow rates in the channels of the spin generators slightly above those predicted by a simple meanfield theory, and a magnetic field with a non-axisymmetric mean part has been observed.

In the following we repeat the above simple estimate of the self-excitation condition for a dynamo module which is no longer a cylinder but a rectangular box. Of course the consideration of box seems less realistic but it opens the possibility of a comparison of the mean-field results with such of direct solutions of the induction equation and to get so an idea on the reliability of the estimates based on the mean-field approach. More specifically, in this way we may learn about the errors resulting from the neglect of induction effects other than the $\alpha$-effect or on the dependence of the results on the number of spin generators.

With this in mind we consider Eq. (17) with constant $\alpha_{\perp}$ in all space and ask for solutions $\overline{\mathbf{B}}$ which are periodic in $X, Y$ and $Z$ with period lengths $2 \pi L_{B_{X}}, 2 \pi L_{B_{Y}}$ and $2 \pi L_{B_{Z}}$, respectively. We may represent $\overline{\mathbf{B}}$ as a sum of a poloidal and a toroidal part,

$$
\overline{\mathbf{B}}=-\nabla \times(\hat{\mathbf{Z}} \times \nabla S)-\hat{\mathbf{Z}} \times \nabla T
$$

with two defining scalars $S$ and $T$. Inserting this in (17) and dropping unimportant constants we find easily that

$$
\begin{array}{r}
\eta \Delta S-\alpha_{\perp} T-\frac{\partial S}{\partial t}=0, \\
\eta \Delta T+\alpha_{\perp} \frac{\partial^{2} S}{\partial Z^{2}}-\frac{\partial T}{\partial t}=0 .
\end{array}
$$

The special solution of (17) which we want to deal with here, is obtained by the ansatz

$$
\begin{aligned}
S & =S_{0} \cos \left(\frac{X}{L_{B_{X}}}\right) \cos \left(\frac{Y}{L_{B_{Y}}}\right) \cos \left(\frac{Z}{L_{B_{Z}}}\right) \exp \{p t\}, \\
T & =\left(T_{0} / S_{0}\right) S,
\end{aligned}
$$

where $S_{0}$ and $T_{0}$ are constants. When inserting this in (22) we arrive at two linear homogeneous equations for $S_{0}$ and $T_{0}$. The requirement that they allow non-trivial solutions leads to

$$
p=-\eta\left(\frac{1}{{L_{B_{X}}}^{2}}+\frac{1}{{L_{B_{Y}}}^{2}}+\frac{1}{{L_{B_{Z}}}^{2}}\right) \pm \frac{\left|\alpha_{\perp}\right|}{L_{B_{Z}}} .
$$

Growing $\overline{\mathbf{B}}$ correspond to the upper sign of the last term of (24) and require that $\left|\alpha_{\perp}\right|$ is sufficiently large. The excitation condition reads

$$
\left|\alpha_{\perp}\right| \geq \eta\left(\frac{L_{B_{Z}}}{{L_{B_{X}}}^{2}}+\frac{L_{B_{Z}}}{L_{B_{Y}}{ }^{2}}+\frac{1}{L_{B_{Z}}}\right) .
$$

Let us now adjust the above solution of the mean-field Eq. (17) to the dynamo module, now assumed to be a rectangular box with the bottom area being a square 
with the side length $L$, and with a height $H$. We require that the edge lengths of the box are just equal to the halves of the corresponding period lengths of the magnetic field. Putting in that sense $\pi L_{B_{X}}=\pi L_{B_{Y}}=L$ and $\pi L_{B_{Z}}=H$ we obtain from (25) the self-excitation condition in the form

$$
\left|\alpha_{\perp}\right| \geq \pi \frac{\eta}{H}\left[1+2\left(\frac{H}{L}\right)^{2}\right] .
$$

We point out that there is, compared to the cylindrical case, one more problematic issue of our reasoning. It consists in the fact that we cannot exclude electric currents through the side walls of our box but only through the bottom and top covers.

For a comparison with case of a cylinder we may assume that the bottom area of the box, $L^{2}$, is equal to that of the cylinder, $\pi R^{2}$. Then (26) can be written in the form (20), but with $2 / \pi$ instead of $(3.83 / \pi)^{2}$. So the results for box and cylinder agree in the limit $H / R \rightarrow 0$, For finite $H / R$, however, the excitation condition derived by considering the box can be easier satisfied than that for the cylinder. Reasons for this discrepancy might be that we are dealing here no longer with axisymmetric fields and that we admit currents through the side walls of the box.

Combining the condition (26) with the relation (18) for $\left|\alpha_{\perp}\right|$ and introducing the edge length $l$ of a Roberts cell by $l=\pi \sqrt{2} L_{U}$ (see Fig. 8a) we find

$$
R_{m \|} R_{m \perp} \phi\left(R_{m \perp}\right) \geq \frac{16 l}{\pi H}\left[1+2\left(\frac{H}{L}\right)^{2}\right] .
$$

Note that the quantities on the left-hand side are determined by $\mathbf{U}$ and $l$ but those on the right-hand side only by the aspect ratios $l / H$ and $L / H$ of an individual spin generator and of the whole dynamo module. When expressing $R_{m \|}$ by a new quantity $R_{m \|}^{*}$ defined by

$$
R_{m \|}=\frac{16 l}{\pi H}\left[1+2\left(\frac{H}{L}\right)^{2}\right] R_{m \|}^{*},
$$

the condition (27) takes the simple form

$$
R_{m \|}^{*} R_{m \perp} \phi\left(R_{m \perp}\right) \geq 1
$$

The geometry of the dynamo module is now hidden in the definition of $R_{m \|}^{*}$. In the Fig. 5 (and likewise in the Figs. 6 and 7) the curve (a) shows the neutral line, or line of marginal stability, in the $R_{m \perp} R_{m \|}^{*}$-plane, which is defined by $R_{m \|}^{*} R_{m \perp} \phi\left(R_{m \perp}\right)=1$. This line separates the region of values of $R_{m \perp}$ and $R_{m \|}^{*}$ allowing dynamo action from that where no dynamo works. As long as the condition (29) applies dynamo action requires that $R_{m \|}^{*}$ exceeds some critical value. It is however possible for any $R_{m \perp}$ if only $R_{m \|}^{*}$ is sufficiently large. 


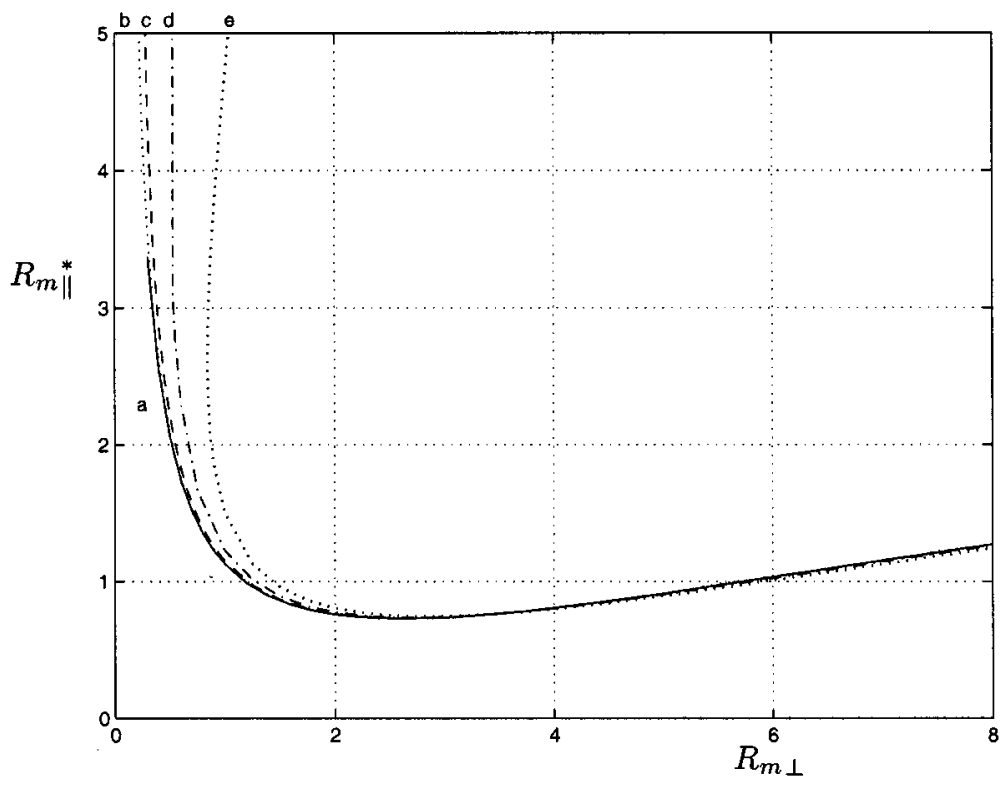

FIGURE 5 Marginal values of $R_{m \|}^{*}$ versus $R_{m \perp}$. Curve (a) is defined by the relation $R_{m \|}^{*} R_{m \perp} \phi\left(R_{m \perp}\right)=1$ obtained in the mean-field approach and already given by Rädler et al. (1996). In the sense of the considerations explained in Section 3.3 it is reproduced in the double limit $L / H \rightarrow \infty$ and $l / H \rightarrow 0$, that is, for $L \gg H \gg l$. The other curves apply also to the limit $L / H \rightarrow \infty$ but to finite $l / H$. For curve (b) the value of $l / \sqrt{2} H$ is $1 / 32$, for (c) $1 / 16$, for (d) $1 / 8$ and for (e) $1 / 5$.

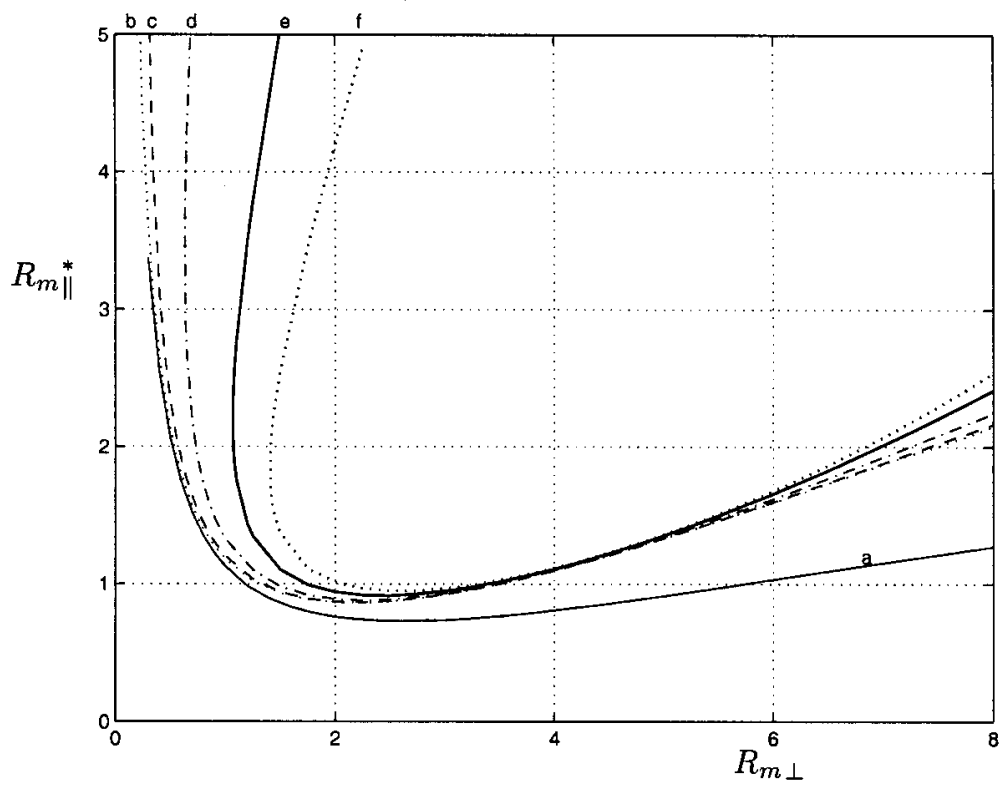

FIGURE 6 Marginal values of $R_{m \|}^{*}$ versus $R_{m \perp}$ for a dynamo box with the aspect ratio $L / H=2$ and different values of $l / H$, or $N$. Curve (a) is the same as in Fig. 5, here given for a comparison only. Curve (b) applies to $N=64$, (c) to $N=32$, (d) to $N=16$, (e) to $N=10$ and (f) to $N=8$. 


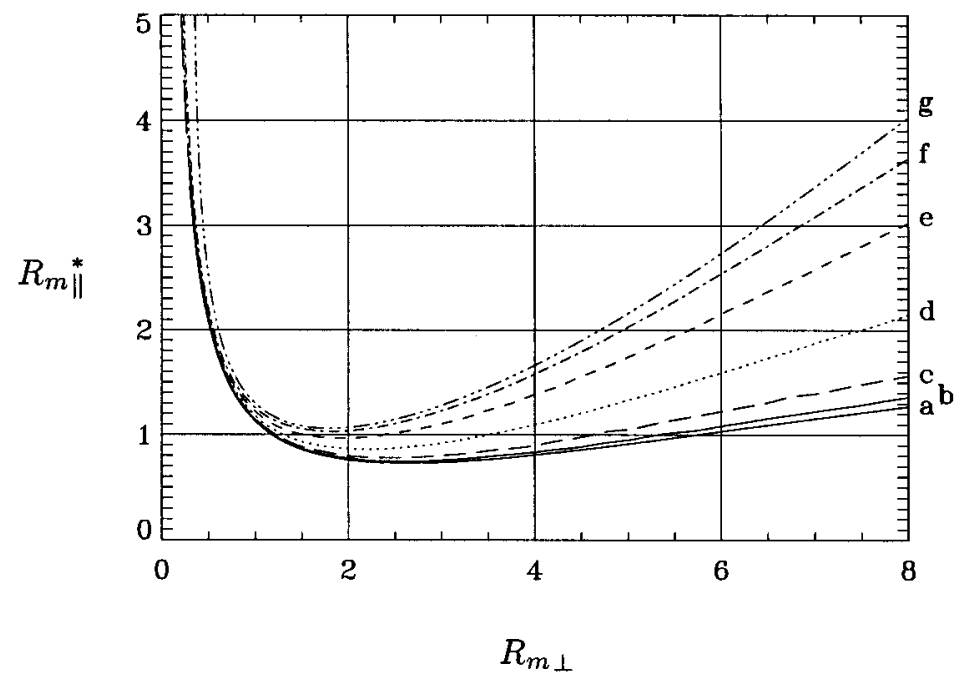

FIGURE 7 Marginal values of $R_{m \|}^{*}$ versus $R_{m \perp}$ for dynamo boxes with different aspect $\operatorname{ratios} L / H$ in the limit $l / H \rightarrow 0$ or, what is the same, $N \rightarrow \infty$. Curve (a) is again the same as in Fig. 5, given for a comparison only. For curve (b) the aspect ratio $L / H$ is 8 , for (c) 4 , for (d) 2 , for (e) 1 , for (f) $1 / 2$ and for (g) $1 / 4$.

\subsection{Comparison of the Results of Mean-Field Approach and Subharmonic Analysis}

Let us now adjust the most easily excitable subharmonic solution $\mathbf{B}$ of the induction Eq. (1) with finite length scales in the same way to the box-shaped dynamo module as we have done it with the mean-field solutions $\overline{\mathbf{B}}$ of the Eq. (17). As explained in Section 2.2 in the parameter range which is of interest for the experiment the most easily excitable solution of that kind is that with $a=b=1$. This means $\pi L_{B}=L$ and $\pi L_{U} / k=H$, where $k$ is now dimensionless. Since for this solution $f_{X}$ and $f_{Y}$ coincide we put $f_{X}=f_{Y}=f$. So the solution to be considered is determined by $f$ and $k$. We have, of course, $f=1 / N$. Using the edge length $l$ of a spin generator, $l=\sqrt{2} \pi L_{U}$, we find $N=\sqrt{2} L / l$ and so $f=l / \sqrt{2} L$. The mentioned relations connecting $L_{B}$ with $L$ and $L_{U} / k$ with $H$ imply $k=L / N H$ and $k=l / \sqrt{2} H$. Apart from a factor, $k$ is just the aspect ratio $l / H$ of an individual spin generator. Instead of $f$ and $k$ we will use in the following also, e.g., $L / H$ and $l / H$.

The period square of $\mathbf{B}$, that is, its two-dimensional periodicity interval in the $X Y$-plane, covers an of area $(2 L)^{2}$, that is, four times the bottom area of the dynamobox. According to (4) this interval contains $2 N^{2}$ cells. Therefore, the box contains $N^{2} / 2$ such cells. In view of the experimental device the best choice of $N$ is $N=10$. Then we have 50 cells in the dynamo-box, which is very close to the real situation with 52 spin generators. An appropriate choice for the aspect ratio $L / H$ of the dynamo box is $L / H=2$. The choice $N=10$ and $L / H=2$ implies that $L / l=10 / \sqrt{2}, k=1 / 5$ and $f=1 / 10$. In the experiment the dynamo worked with values of $R_{m \perp}$ between 1.2 and 2.0 .

Analogous to the mean-field approach we then interpret the excitation condition of the solution $\mathbf{B}$ specified in this way as the excitation condition of the experimental device. Again we have to point out that there are reasons to consider this interpretation with caution. As in the case of the mean-field approach we cannot exclude currents intersecting the walls of the box, now not even at the bottom and the top surfaces. 
In addition the cells of the flow pattern, or the spin generators, are necessarily cut by the side walls of the dynamo box, see also Fig. 8 , in the best case by at least two of them.

We will represent our results again in the form of marginal curves in the $R_{m \perp} R_{m \|^{-}}^{*}$ plane, with $R_{m \|}^{*}$ again defined by (28).

Let us first consider the limit $L / H \rightarrow \infty$. If then in addition $l / H \rightarrow 0$ we obtain the same marginal curve in the $R_{m \perp} R_{m \|}^{*}$-diagram as in the mean-field approach, that is, curve (a) in Fig. 5 (and likewise in the Figs. 6 and 7). In other words, it is the condition

$$
L \gg H \gg l,
$$

which brings us back to the mean-field approach.

It is of some interest to consider the limit $L / H \rightarrow \infty$ also for finite $l / H$. Figure 5 shows results for $l / \sqrt{2} H=1 / 32,1 / 16,1 / 8$ and $1 / 5$. At this point we recall that in our simple mean-field approach we neglected all contributions to the electromotive force $\mathcal{E}$ connected with derivatives of $\overline{\mathbf{B}}$, which would give rise to the introduction of a mean-field conductivity different from the usual conductivity etc. In the calculation of the coefficient $\alpha_{\perp}$ no derivatives of $\overline{\mathbf{B}}$ are considered either. The deviations of the results for finite $l / H$ from that for $l / H \rightarrow 0$ reflect just the influence of contributions to $\mathcal{E}$ connected with $Z$-derivatives of $\overline{\mathbf{B}}$ neglected in our mean-field approach. For the considered values of $l / H$ and $R_{m \perp}>2$ this influence is very weak. For smaller $R_{m \perp}$, however, it is well important. At least for $l / \sqrt{2} H=1 / 5$ it can be clearly seen that dynamo action requires that not only $R_{m \|}^{*}$ but also $R_{m \perp}$ exceeds a critical value.

Let us now proceed to finite $L / H$ and finite $l / H$. We fix the aspect ratio of the dynamo box first by $L / H=2$. Then we have $l / H=2 \sqrt{2} / N$. In Fig. 6 neutral curves in the $R_{m \perp} R_{m \|}^{*}$-plane are given for $N=64,32,16,10$ and 8 . At least for $N=16,10$ and 8 it is obvious that a dynamo can only work if both $R_{m \|}^{*}$ and $R_{m \perp}$ exceed critical values. Again the deviations of the results presented here from that of our mean-field estimate reflect the influence of the derivatives of $\overline{\mathbf{B}}$ whose contribution to $\mathcal{E}$ has been neglected in this estimate. Now, however, not only the derivatives with respect to $Z$ are of interest but also those with respect to $X$ and $Y$. The values of $R_{m \|}^{*}$ are always higher than those obtained in the mean-field approximation. The deviations are no longer restricted to a range of small $R_{m \perp}$ but occur also for large $R_{m \perp}$. For the situation in the experiment, $N=10$ and $R_{m \perp}$ between 1.2 and 2.0, the relative deviation is larger than $20 \%$. In the limit $N \rightarrow \infty$, the neutral curves coincide but are different from curve (a), at least for $R_{m \perp}>1$. Here the condition $L \gg H$ contained in (30) is not satisfied.

We finally compare dynamo boxes with different aspect ratios $L / H$ in the limit $l / H \rightarrow 0$. Since $l / H=\sqrt{2}(L / H) / N$, this limit is for any fixed $L / H$ equivalent with $N \rightarrow \infty$. Figure 7 shows results for $L / H=8,4,2,1,1 / 2$ and $1 / 4$. As mentioned before in (30), the results for large $L / H$ and $H / l$ coincide with those of the meanfield approach.

\section{CONCLUSIONS}

We have dealt with the Roberts dynamo in two different ways. Firstly, we reduced the induction equation governing the magnetic field, considering in particular 
subharmonic solutions, to a matrix eigenvalue problem and solved it numerically. Secondly, we gave analytic solutions of an equation for the mean magnetic field, which can be derived under certain assumptions from the induction equation. Both kinds of solutions have been used for estimates of the self-excitation condition of the Karlsruhe dynamo experiment. We focussed particular attention to the neutral lines in the plane of the two Reynolds numbers $R_{m \|}$ and $R_{m \perp}$ which separate the region of these parameters allowing dynamo action from the other where dynamos cannot work.

In our simple mean-field approach there occurs a critical value of $R_{m \|}$ so that a dynamo is only possible if $R_{m \|}$ exceeds this value, but dynamo action may take place with an arbitrary value of $R_{m \perp}$ if only $R_{m \|}$ is sufficiently large. In the approach based on subharmonic solutions of the induction equation, however, critical values in that sense occur, at least for a not too high number of spin generators in the dynamo module, for both $R_{m \|}$ and $R_{m \perp}$.

Apart from this, the requirements for dynamo action derived with our mean-field approach are lower than those concluded from the subharmonic solutions, that is, in the first case the self-excitation conditions can be satisfied with smaller values of $R_{m \|}$ and $R_{m \perp}$ than in the second one.

There are good reasons to believe that this discrepancy is due to the neglect of induction effects in our mean-field estimate, in particular the effects which are usually described in terms of a mean-field conductivity. That is why we consider the predictions made on the basis of the subharmonic solutions as more realistic than the mean-field estimates.

Of course, both kinds of predictions have to be considered with caution, e.g., because the solutions used of the original induction equation as well as those of the corresponding mean-field equation do not satisfy realistic boundary conditions.

It is, of course, not possible to derive detailed predictions concerning the experiment with a cylindrical dynamo module from our considerations on a rectangular dynamo box. We can only conclude from the above results in what sense the meanfield results for a cylindrical dynamo module will change if, e.g., the finite number of spin generators is taken into account. A feature that should not critically depend on the form of the dynamo-active region is the shape of the neutral line in the diagram of the axial and helical flow rates. This line, in particular its slope, obtained in the subharmonic approach reflects the experimental data clearly better than the corresponding result of the simple mean-field approach (see Rädler et al., 2001).

The subharmonic formalism for the solution of the induction equation, which we established in Section 2.1, can save much computation time when one is interested in a specific spatial period of the B-field only. As we will show in Appendix B this formalism may also help to interpret results of direct numerical simulation of $\mathcal{A B C}$ dynamos as the ones reported by Galanti et al. (1992).

\section{Acknowledgements}

This work has been done during a stay of F.P. at the Astrophysikalisches Institut Potsdam as a guest scientist. The authors are grateful to M. Reinhardt for fruitful discussions and for the translation of the paper by Arnold and Korkina (1983). 


\section{References}

Arnold, V.I. and Korkina, E.I., "The growth of a magnetic field in a three-dimensional steady incompressible flow", Vest. Mosk. Un. Ta. Ser. 1, Matem. Mekh. 3, 43-46 (1983).

Busse, F.H., Müller, U., Stieglitz, R. and Tilgner, A., "A two-scale homogeneous dynamo: an extended analytical model and an experimental demonstration under development", Magnetohydrodynamics 32, 235-248 (1996)

Childress, S., "Alpha-effect in flux ropes and sheets", Phys. Earth Planet. Int. 20, 172-180 (1979).

Childress, S. and Gilbert, A.D., Stretch, Twist, Fold: The Fast Dynamo, Springer (1995).

Dombre, T., Frisch, U., Greene, J.M., Hénon, M., Mehr, A. and Soward, A.M., "Chaotic streamlines in the ABC flows", J. Fluid Mech. 167, 353-391 (1986).

Feudel, F., Seehafer, N. and Schmidtmann, O., "Fluid helicity and dynamo bifurcations", Phys. Lett. A 202, 73-78 (1995).

Galanti, B., Sulem, P.-L. and Pouquet, A., "Linear and nonlinear dynamos associated with ABC flows", Geophys. Astrophys. Fluid Dynam. 66, 183-208 (1992).

Galanti, B., Pouquet, A. and Sulem, P.-L., "Influence of the period of an ABC flow on its dynamo action", In: Solar and Planetary Dynamos (Eds. M.R.E. Proctor, P.C. Matthews and A.M. Rucklidge) pp. 99-104, Cambridge University Press (1993).

Galloway, D.J. and Frisch, U., "A numerical investigation of magnetic field generation in a flow with chaotic streamlines", Geophys. Astrophys. Fluid Dynam. 29, 13-18 (1984).

Galloway, D.J. and Frisch, U., "Dynamo action in a family of flows with chastic streamlines", Geophys. Astrophys. Fluid Dynam. 36, 53-83 (1986).

Gilbert, A.D. and Childress, S., "Evidence for fast dynamo action in a chaotic web", Phys. Rev. Lett. 65, 2133-2136 (1990).

Krause, F. and Rädler, K.-H., Mean-Field Magnetohydrodynamics and Dynamo Theory, Pergamon Press (1980).

Müller, U. and Stieglitz, R., "Can the Earth's magnetic field be simulated in the laboratory?", Naturwissenschaften 87, 381-390 (2000).

Plunian, F., Marty, Ph. and Alemany, A., "Kinematic dynamo action in a network of screw motions. Application to the core of a fast breeder reactor", J. Fluid Mech. 382, 137-154 (1999).

Rädler, K.-H., Apel, A., Apstein, E. and Rheinhardt, M., "Contributions to the Theory of the Planned Karlsruhe Dynamo Experiment", Report Astrophysical Institute Potsdam (1996).

Rädler, K.-H., Apstein, E., Rheinhardt, M. and Schüler, M., "Contributions to the Theory of the Planned Karlsruhe Dynamo Experiment - Supplements and Corrections", Report Astrophysical Institute Potsdam (1997a).

Rädler, K.-H., Apstein, E. and Schüler, M., "The Alpha-Effect in the Karlsruhe Dynamo Experiment", Proc. 3rd Int. Conf. on Transfer Phenomena in Magnetohydrodynamics and Electro-conducting Flows, Vol. I, Aussois, France, pp. 9-14 (1997b).

Rädler, K.-H., Apstein, E., Rheinhardt, M. and Schüler, M., "The Karlsruhe Dynamo Experiment - A MeanField Approach", Studia Geophysica et Geodaetica 42, 302-308 (1998).

Rädler, K.-H., Rheinhardt, M., Apstein, E. and Fuchs, H., "On the mean-field theory of the Karlsruhe Dynamo Experiment" accepted in Nonlinear Processes in Geophysics (2001).

Roberts, G.O., "Dynamo action of fluid motions with two-dimensional periodicity", Phil. Trans. R. Soc. Lond. A 271, 411-454 (1972).

Soward, A.M., "Fast dynamo action in a steady flow", J. Fluid Mech. 180, 267-295 (1987).

Soward, A.M., "On dynamo action in a steady flow at large magnetic Reynolds number", Geophys. Astrophys. Fluid Dynam. 49, 3-22 (1989).

Soward, A.M., "A unified approach to a class of slow dynamos", Geophys. Astrophys. Fluid Dynam. 53, 81-107 (1990).

Soward, A.M., "Fast Dynamos", In: Lectures on Solar and Planetary Dynamos (Eds. M.R.E. Proctor and A.D. Gilbert) pp. 181-217, Cambridge University Press (1994).

Stieglitz, R. and Müller, U., "Experimental demonstration of a homogeneous two-scale dynamo", Phys. Fluids 13(3), 561-564 (2001).

Tilgner, A., “A kinematic dynamo with a small scale velocity field”, Phys. Lett. A 226, 75 (1997).

Tilgner, A. and Busse, F.H., "Subharmonic dynamo action of fluid motions with two-dimensional periodicity", Proc. R. Soc. Lond. A 448, 237-244 (1995). 


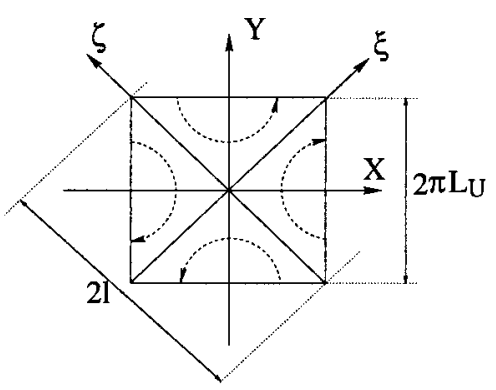

(a)

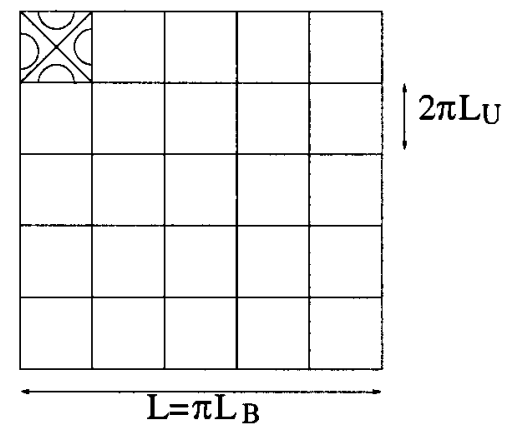

(b)

FIGURE 8 (a) A periodic square of the Roberts flow covering an area $\left(2 \pi L_{U}\right)^{2}$ corresponding to two cells which contains two Roberts cells. (b) The parallelepipedal dynamo box contains 25 periodic squares $2 \pi L_{U} \times 2 \pi L_{U}$ or equivalently 50 Roberts cells. Therefore, $N=L_{B} / L_{U}=10$.

\section{APPENDIX A}

Our representation (2) of the Roberts flow as well as our representation (18) of the coefficient $\alpha_{\perp}$ differ from those in Rädler et al. (1996, 1997b, 1998) (referred to as R96-98 in the following). We explain here the relations between these representations.

In R96-98 the Roberts flow is given in the form

$$
\begin{aligned}
& u_{\xi}=u_{\perp} \frac{\pi}{2} \sin \left(\frac{\pi}{l} \xi\right) \cos \left(\frac{\pi}{l} \zeta\right), \\
& u_{\zeta}=-u_{\perp} \frac{\pi}{2} \cos \left(\frac{\pi}{l} \xi\right) \sin \left(\frac{\pi}{l} \zeta\right), \\
& u_{Z}=u_{\|}\left(\frac{\pi}{2}\right)^{2} \sin \left(\frac{\pi}{l} \xi\right) \sin \left(\frac{\pi}{l} \zeta\right),
\end{aligned}
$$

with respect to a coordinate system $(\xi, \zeta, Z)$ whose relation to the system $(X, Y, Z)$ is shown in Fig. 8. With a coordinate transformation

$$
X=\frac{1}{\sqrt{2}}(\xi-\zeta), \quad Y=\frac{1}{\sqrt{2}}(\xi+\zeta)
$$

and the substitutions

$$
l=\pi L_{U} \sqrt{2}, \quad U=\frac{\pi}{2 \sqrt{2}} u_{\perp}, \quad 2 \chi U=\left(\frac{\pi}{2}\right)^{2} u_{\|}
$$

we rediscover our representation (2).

The result for $\alpha_{\perp}$ occurs in R96-98 in the form 


$$
\alpha_{\perp}= \pm \frac{\pi^{2}}{16} \frac{\eta}{l} R_{m \|} R_{m \perp} \phi\left(R_{m \perp}\right), \quad R_{m \perp}=\frac{l u_{\perp}}{2 \eta}, \quad R_{m \|}=\frac{l u_{\|}}{\eta} .
$$

Starting from this and using (A.4) we arrive at (18) and (19).

By the way, we see here that the Roberts flow pattern has two different period length, $2 l$ and $2 \pi L_{U}$. According to (A.4) their ratio is irrational.

\section{APPENDIX B}

The $\mathcal{A B C}$ flow, named after Arnold, Beltrami and Childress, is periodic in the three cartesian directions and has been studied in details in the frame of fast dynamo theory (for references on $\mathcal{A B C}$ dynamo see e.g., Arnold and Korkina 1983; Galloway and Frisch 1984, 1986; Dombre et al., 1986; Gilbert and Childress, 1990; Feudel et al., 1995). In the paper by Galanti et al. (1992) (subsequently denoted G92), the authors considered the $\mathcal{A B C}$ dynamo with a magnetic field satisfying (4) with $2 \pi L_{B}$ being the period of the magnetic field identical in the three cartesian directions (see also Galanti et al., 1993). They found that the magnetic modes with a larger period length than the flow pattern, that is $N>1$, can be more easily excited than those with $N=1$. Furthermore, they found that there exist values of $R_{m}$ for which dynamo action is possible for $N=2$ but not for $N=1$.

At first glance, these results seem to be in conflict with our explanations in Section 2.1 and the numerical results presented in Section 2.2 according to which the largest growth rates for the magnetic modes belonging to a given $N$ are independent of $N$. The difference in the results is connected with the difference in the length scales, which are responsible for the dissipation. In the $\mathcal{A B C}$ case the largest length scale of the magnetic fields is $N$ times that of the flow. In the Roberts case, however, the largest length scales are those in the $X$ and $Y$ directions, which are infinite. By the way, infinite length scales in the $\mathcal{A B C}$ case would imply a homogeneous magnetic field, which cannot decay. Admitting this we would no longer deal with a self-exciting dynamo but with an externally excited one. In the Roberts case the parts of the magnetic field with infinite length scales still depend on $Z$ and are therefore subject to dissipation.

Some results of G92 can be understood in the light of the subharmonic formalism explained in Section 2.1. For example, in Fig. 10 of G92, the growth rates of different subharmonic modes are given for $N=2$ ( $N$ is denoted $k_{0}$ in G92). We can identify these growth rates as corresponding to different independent solutions. According to our classification (Table I), for $N=2$ there exist four independent solutions characterized by

$$
(a, b, c) \in\{(0,0,0),(0,0,1),(0,1,1),(1,1,1)\} .
$$

The curves of Fig. 10b of G92 correspond to $(0,0,0)$. The curves $1-3$ of Figs. 10c and 4-9 of Fig. 10d correspond to $(0,0,1)$. And the curves $4-6$ of Fig. 10c and 1-3 of Fig. 10d correspond to $(0,1,1)$. Therefore, it is clear why the curves of each group exhibit the same growth rate. Some further remarks given in the text of G92, p. 197, can also be interpreted in terms of our independent solutions. 